\title{
Baby mit Blessuren: War Gewalt im Spiel?
}

\begin{abstract}
Wenn Babys oder Kleinkinder, die noch nicht laufen, unerklärliche Blutergüsse aufweisen, sollten die Warnlampen angehen: Solche Blessuren können darauf hinweisen, dass die Kinder misshandelt werden.
\end{abstract}

- Ärzte einer pädiatrischen Klinik in Wisconsin sind 400 Fällen nachgegangen, die im Alter von maximal einem Jahr dem „Child Protection Team“ der Klinik vorgestellt worden waren; es sollte der Verdacht einer aktuellen Misshandlung geklärt werden. Von den 200 Kindern, bei denen sich dieser Verdacht bestätigte, hatte mehr als ein Viertel eine geringfügige, aber deutlich sichtbare Verletzung in der Vorgeschichte, die mindestens einem Elternteil aufgefallen war und für die sich keine plausible Erklärung fand. In $80 \%$ handelte es sich dabei um Blutergüsse oder Striemen an Kopf, Gliedmaßen oder Stamm, in 11\% um eine Verletzung in der Mundhöhle, z. B. ein eingerissenes Frenulum oder eine Verletzung der Zunge. Derartige anamnestisch erfasste Blessuren wies keines der Kinder einer Vergleichsgrup-

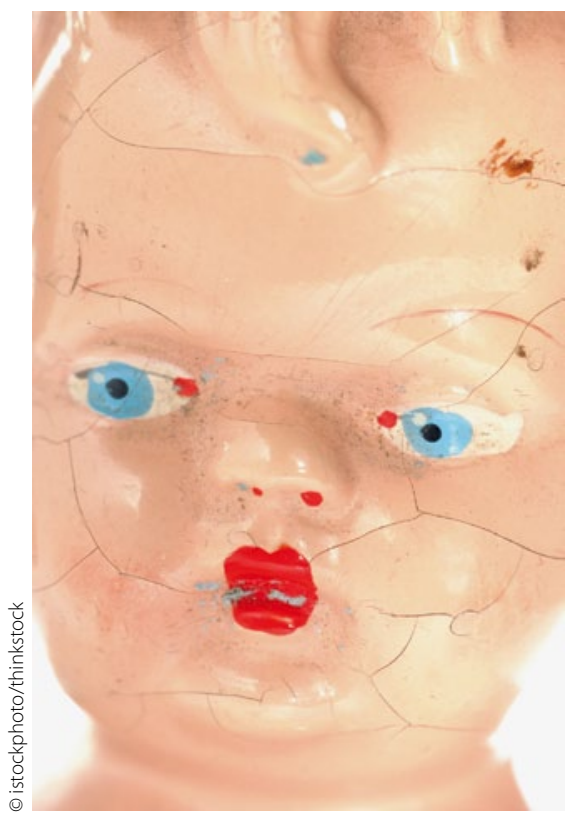

Schweren Misshandlungen gehen oft "geringfügige" Verletzungen voraus. pe auf, bei denen zwar der Verdacht auf eine Misshandlung bestand, diese aber ausgeschlossen werden konnte.

\section{Vorboten für schlimme Gewalttaten}

Bei Kindern, die noch nicht im Laufalter sind, sei es unwahrscheinlich, dass sie sich solche Verletzungen selbst zufügten, argumentieren Lynn K. Sheets und Kollegen vom Medical College of Wisconsin. Die Forscher werten die geringfügigen Blessuren ohne erkennbare Ursache daher als „Vorboten“ für die später erfolgte schwerere Misshandlung. Bei 63 der untersuchten Kinder fanden sich solche verdächtigen Läsionen in der Vorgeschichte, zum allergrößten Teil (95\%) waren diese in den ersten sieben Lebensmonaten aufgetreten. 55 Kinder mit solchen primären Blessuren waren später Opfer einer schwereren Misshandlung geworden.

Wann immer sich bei Kleinkindern, die noch nicht laufen können, keine plausible Erklärung findet für blaue Flecken, Blutergüsse, Striemen oder auch Verletzungen in der Mundhöhle, sollte man an Missbrauch denken, legen Sheets et al. ihren Kollegen ans Herz. Schon eine einzelne Läsion könne ein Vorbote für eine schlimmere Gewalttat sein. Die Studie habe gezeigt, so die Autoren, dass es sich häufig um Wiederholungstaten handelt. Wer die genannten Anzeichen ernst nehme und schon den ersten begründeten Verdacht melde, könne demnach schlimmere Fälle von Kindesmisshandlung verhindern.

DR. ELKE OBERHOFER -

\footnotetext{
- Sheets L. K. et al Sentinel Injuries in Infants Evaluated for Child Physical Abuse. Pediatrics 2013; online March 11, 2013; doi: 10.1542/
} peds.2012-2780

\section{Achtung:}

\section{Hier muss der Dummy durch eine Anzeige ersetzt werden !!}

